\title{
MPO G-463A Gene Polymorphism and Circulating Matrix Metalloproteinase-9, Myeloperoxidase Levels in Coronary Artery Disease Patients
}

\author{
Yusuf Dogus (Corresponding author) \\ Department of Biochemistry, Faculty of Medicine, \\ Cukurova University, Adana, Turkey \\ E-mail: yusufdogus2@gmail.com \\ Necip Ilhan \\ Department of Biochemistry, Faculty of Medicine, \\ Firat University, Elazig, Turkey \\ Dilara Kaman \\ Department of Biochemistry, Faculty of Medicine, \\ Firat University, Elazig, Turkey \\ Sertap Dogus \\ Public Health Service Department, \\ Provincial Directorate of Health, Adana, Turkey \\ Mehmet Akbulut \\ Department of Cardiology, Faculty of Medicine, \\ Firat University, Elazig, Turkey
}

\begin{abstract}
Objective: Coronary artery disease(CAD) patients living in Elazı̆̆ region is to assess the levels of matrix metalloproteinase-9(MMP-9) and myeloperoxidase(MPO), polymorphisms MPO gene.

Research Design and Methods: Eighty-eight patients with angiographically diagnosed coronary artery disease between the ages of 18-80 who applied to Department of Cardiology, Faculty of Medicine, Firat University were enrolled in this study. MPO, MMP-9 levels in plasma samples of individuals were determined by enzyme-linked immuno sorbent assay (ELISA) according to the kit procedure. MPO polymorphism was studied using the PCR-RFLP technique.
\end{abstract}

Results: When plasma MPO and MMP-9 levels were compared in the control and CAD groups; MPO levels were statistically significant ( $\mathrm{p}<0.05)$. MMP-9 levels were not statistically significant. MPO gene polymorphism was not found statistically significant in CAD.

Conclusion: MPO gene polymorphism is thought to be associated with greater population populations in $\mathrm{CAD}$ and may be used in the future as a biomarker for plasma MPO enzyme levels in CAD.

Key words: MPO, coronary artery disease, MMP-9

DOI: $10.7176 / \mathrm{JHMN} / 83-02$

\section{Introduction}

Oxidative stress plays an important role in the initiation and progression of cardiovascular disease (1). Oxidative stress biomarkers have been identified in coronary artery disease (CAD) (2-4). CAD has a multifactorial etiology, and it is thought to result from an interaction between genetic background and environmental factors (5-7).

Myeloperoxidase (MPO) is a potent oxidative enzyme and it is expressed by neutrophils, monocytes, and some populations of macrophages. It catalyzes the modification of LDL in atherogenesis (8). MPO 
has an important role in infectious diseases and in CAD (9). Several studies have shown that there is an association between MPO levels and CAD (8). Levels of MPO activity is associated with various genetic polymorphisms so that associated with cardiovascular risk (10). It has been shown that elevated MPO levels are associated with coronary artery disease (CAD) (11).

The gene coding for human myeloperoxidase, is located in chromosome 17q23.1. A few polymorphisms are described for the MPO gene that affects its transcription (12). In this polymorphism, the presence of a $\mathrm{G}$ rather than $\mathrm{A}$ at position $463 \mathrm{bp}$ gives rise to three different genotypes: AA, AG and GG. To date, a number of studies have been suggested the potential association between the MPO G-463A polymorphism and CAD. However the results were inconsistent. Some of this studies have shown that GG genotype is a significant predictor of CAD and endothelial dysfunction when compared to AA/AG genotypes (13-16).

Nikpoor et all., showed that individuals with A allele has a lower activity of the MPO and so that it leads to lower oxidation of LDL, therefore A allele of the MPO genotype has a protective role in the occurrence of CAD (13). Nevertheless, there are conflicting data on the relation between MPO levels and its polymorphism with CAD $(17,18)$.

Matrix metalloproteinases (MMPs) are a family of zinc-containing endoproteinases which mainly secreted by monocyte-derived macrophage and vascular smooth muscle cells (19). MMP-9 is the most studied enzyme in the MMP-family. It is a key enzyme which determinant of extracellular matrix degradation, and collagen is the main substrate of MMP-9. MMP-9 concentrations and activity was higher in human atherosclerotic plaques (20-23). The serum level of MMP-9 might be a sensitive inflammation marker (24) and a predictor of cardiovascular mortality in patient with CAD (25).

The purpose of this study was to test the hypothesis that MPO polymorphism $-463 \mathrm{~A} / \mathrm{G}$ is associated with CAD. In addition, as secondary objective we also investigated the association between CAD and MPO, MMP-9 plasma levels. Oxidative stress parameters have been evaluated in CAD frequently. However, there has not been much published work related to oxidative stress in CAD patients in Turkey. Similarly, not much literature is available on myeporexoidase and MMP-9 level; MPO gene polymorphism and its association with lipids in CAD patients in Turkey. We, therefore evaluated these parameters in CAD patients.

\section{Materials and Methods}

The patient population and documentation of CAD severity

This was a case-control study. The cases and controls were the angiographically confirmed. The study sample comprised 176 persons who underwent coronary angiography for diagnostic purposes. The angiograms were assessed by two cardiologists who were unaware that the patients were to be included in the study. The three main arteries (left anterior descending artery, left circumflex artery, or right coronary artery) were classified as free of disease, or presenting $\geq 50 \%$ stenosis. Patients having a normal angiogram with no atherosclerosis or lesions in coronary arteries were considered as CAD control subjects $(\mathrm{n}=88)$. (Table 1$)$. Written informed consent was obtained from all subjects, and a local ethical committee approved the study protocol.

\section{Blood sample collection}

Patients were fasted for at least $6 \mathrm{~h}$ before venous blood samples were drawn into $5 \mathrm{~mL}$ EDTA vacuum tubes and blood samples were separated in a refrigerated centrifuge within $15 \mathrm{~min}$ of collection for determination of plasma MPO and MMP-9. Plasma was divided into small aliquots, stored at $-20^{\circ} \mathrm{C}$ until analysis. For determination of serum lipids, a blood sample was obtained from the cubital vein of each participant and the samples were sent for detection within $1 \mathrm{~h}$. by using the Advia 1800 (Siemens Diagnostics, Tarrytown, NY, USA) autoanalyzer and reactives.

\section{Determination of plasma levels of MPO and MMP-9}

Plasma levels of MPO (Boster Biological Technology Co.,Ltd. USA; Cat no: EK0850) and MMP-9 (Boster Biological Technology Co.,Ltd. USA; Cat no: EK0465) were measured by enzyme-linked immunosorbent assay according to kit protocols.

\section{Genotyping}

DNA was extracted from EDTA anti-coagulated whole blood from all subjects (controls and patients) and was prepared from DNA isolation kit (Sigma-Aldrich, GenElute Blood genomic DNA Kit, Catalog no: NA2010, Germany). The MPO 463 -A/G polymorphism was genotyped by restriction fragment length polymorphism (RFLP) assay. The polymorphic site at position -463 of the MPO gene was 
amplified with use of forward primer (50-CGG TAT AGG CAC ACA ATG GTG AG-30) and reverse primer (50-GCA ATG GTT CAA GCG ATT CTT C-30) as described in the literature.

Amplification was carried out in a volume of $20 \mu$ containing $1-10 \mu \mathrm{g}$ of genomic DNA, 10 pmol of each primer, $0.5 \mathrm{mM}$ of each dNTP, reaction buffer, and 1.0 U of Taq DNA polymerase. Amplified products were digested overnight with Aci I at $37{ }^{\circ} \mathrm{C}$. The resulting fragments were 289 bp for $A / A$, $289,169,120 \mathrm{bp}$ for A/G and allele and 169, $120 \mathrm{bp}$ for $G G$ genotype.

\section{Statistical analysis}

All of the statistical analyses were performed by using the SPSS 12.0 statistical package. We have presented normally distributed data as mean \pm S.D. Categorical variables are presented by frequency of counts, and inter group comparisons analysed by a chi-squared analysis. We compared cases and controls on biochemical parameters and MPO levels using Student's t-test. Anova test was used to investigate the plasma lipid, MPO and MMP-9 levels between the genotypes. When we analyzed the quantitative relationships between MPO and MMP-9, bivariate

correlation coefficients were calculated, using Pearson's for parametric data. For the comparisons of the values of different groups, $\mathrm{P}<0.05$ was considered statistically significant.

\section{Results}

\section{Clinical characteristics of patients with and controls:}

The clinical characteristics of the patients and controls are shown in Table 1. Total cholesterol and LDL cholesterol levels were significantly higher in patients ( $p<0.0001$ and $p<0.01$ respectively). Levels of triglyceride and HDL cholesterol were not significantly different among groups. Sistolic blood pressure and the fregquency of smoking were higher in patients $(\mathrm{p}<0.05, \mathrm{p}<0.01$, respectively). MPO level was higher in CAD patients $(\mathrm{p}<0.05)$ although MMP-9 level was not significantly different among groups (figure 1).

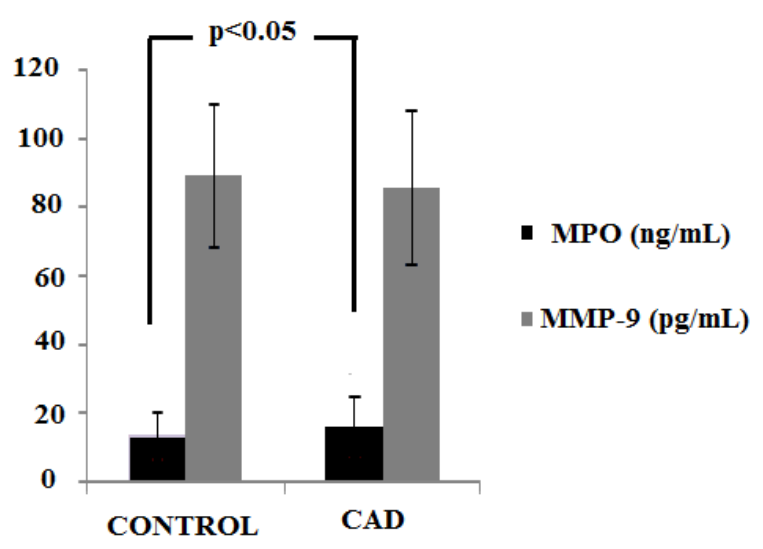

Figure 1. Plasma MPO and MMP-9 levels in control and CAD patients.

Table 1. Clinical characteristics of individuals with CAD and controls

\begin{tabular}{|c|c|c|c|}
\hline & Control (n:88) & $\mathrm{CAD}(\mathrm{n}: 88)$ & $\mathrm{p}$ \\
\hline Gender (F/M) & $43 / 45$ & $34 / 54$ & 0,224 \\
\hline Age (years) & $50,21 \pm 11,41$ & $62,67 \pm 11,81$ & $0,000^{* *}$ \\
\hline Smoking, (\%) & $(\% 87,8)$ & $\% 38,59 \quad(\% 61,41)$ & $0,008^{*}$ \\
\hline Systolic blood pressure (mmHg) & $119,84 \pm 15,43$ & $126,84 \pm 23,84$ & $0,032^{*}$ \\
\hline Diastolic blood pressure $(\mathrm{mmHg})$ & $74,84 \pm 11,48$ & $79,47 \pm 10,42$ & 0,332 \\
\hline Total cholesterol (mg/dL) & $175,44 \pm 51,17$ & $206,54 \pm 51,01$ & $0,000^{* *}$ \\
\hline HDL-cholesterol (mg/dL) & $45,24 \pm 9,10$ & $42,41 \pm 14,84$ & 0,130 \\
\hline LDL-cholesterol (mg/dL) & $114,83 \pm 44,16$ & $132,44 \pm 40,64$ & $0,007^{*}$ \\
\hline VLDL-cholesterol (mg/dL) & $32,40 \pm 27,64$ & $31,75 \pm 22,76$ & 0,529 \\
\hline
\end{tabular}




\section{Genotype frequencies}

Genotypic and allelic frequencies for the study groups are shown in Table 2. No statistically significant difference found in the distribution of the MPO $-463 \mathrm{G} / \mathrm{A}$ genotye and allele fequency in the patients and control groups.

Table 2. Genotype and allele frequencies among groups

\begin{tabular}{|l|c|c|}
\hline & $\begin{array}{c}\text { CONTROL } \\
\mathbf{n : 8 8}\end{array}$ & $\begin{array}{c}\text { CAD } \\
\mathbf{n : 8 8}\end{array}$ \\
\hline Genotype & & \\
GG Genotype & $63(\% 71,6)$ & $64(\% 72,7)$ \\
AG Genotype & $23(\% 26,1)$ & $21(\% 23,9)$ \\
AA Genotype & $2(\% 2,3)$ & $3(\% 3,4)$ \\
Allele & & $\% 84,66$ \\
G Allele & $\% 84,66$ & $\% 15,34$ \\
\hline A Allele & $\% 15,34$ & \\
\hline
\end{tabular}

\section{Association of the MPO gene polymorphism with lipids, MPO and MMP-9 levels.}

Table 3 shows the association between the MPO gene polymorphism and lipid levels, Figure 2 shows the association between the MPO gene polymorphism and MPO, MMP-9 levels in controls and patiens with CAD, respectively. The higher total cholesterol, LDL-cholesterol and HDL cholesterol level was shown in the AA genotype in controls and patients but this was not statistically significant. No statistically significant difference found in the levels of MPO and MMP-9 among genotypes of MPO in controls and patients with CAD.

Table 3. Lipid levels of the study subjects according to MPO genotypes in controls and patients.

\begin{tabular}{lcccccc}
\hline \multicolumn{3}{c}{ CONTROL } & \multicolumn{3}{c}{ CAD } \\
\hline & GG (n:63) & AG (n:23) & AA (n:2) & GG (n:64) & AG (n:21) & AA (n:3) \\
Total cholesterol (mg/dL) & $171,82 \pm 48,7$ & $180,95 \pm 57,01$ & $226,00 \pm 48,08$ & $207,26 \pm 55,28$ & $203,76 \pm 40,55$ & $210,66 \pm 19,21$ \\
HDL-cholesterol (mg/dL) & $45,13 \pm 9,52$ & $44,47 \pm 7,26$ & $57,50 \pm 10,60$ & $43,06 \pm 14,95$ & $40,99 \pm 15,77$ & $38,56 \pm 1,25$ \\
LDL-cholesterol (mg/dL) & $112,70 \pm 42,8$ & $118,14 \pm 49,37$ & $144,00 \pm 4,24$ & $132,29 \pm 41,53$ & $131,71 \pm 41,38$ & $140,66 \pm 17,78$ \\
VLDL-cholesterol (mg/dL) & $32,84 \pm 30,16$ & $32,04 \pm 20,91$ & $23,00 \pm 16,97$ & $31,63 \pm 25,29$ & $30,19 \pm 11,41$ & $45,33 \pm 28,04$ \\
\hline
\end{tabular}
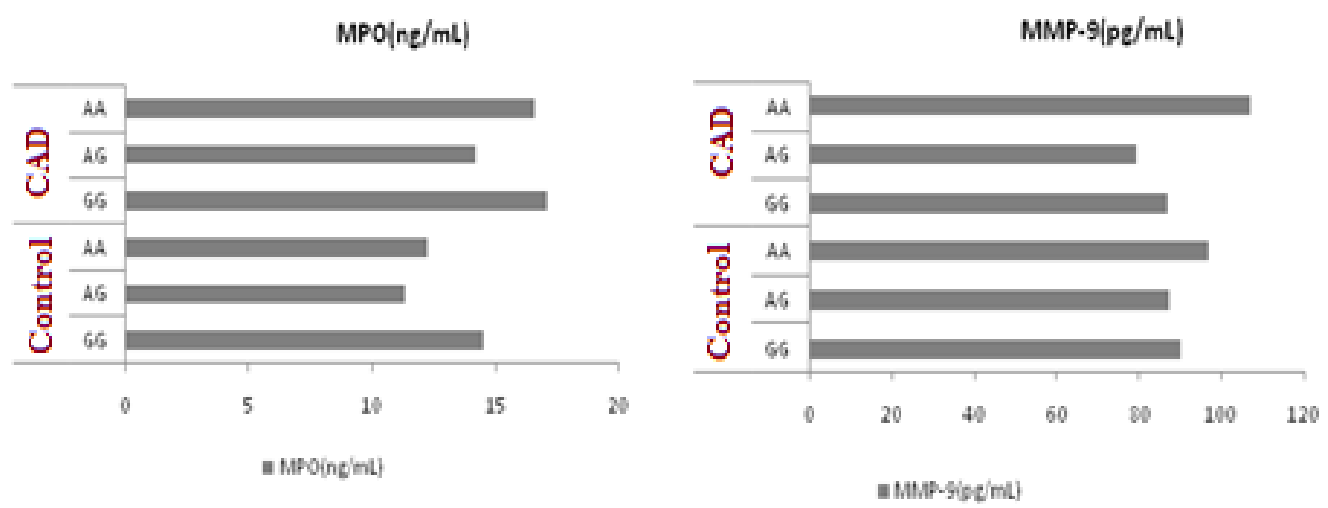

Figure 2. MPO and MMP-9 levels of the study subjects according to MPO genotypes in controls and patients. 
in this study we found that in healthy groups with $G$ allele had elevated MPO levels than A allele $(p<0.05)$ and among subjects with $G$ alleles, CAD patients with $G$ allele had higher level of MPO compared with controls $(\mathrm{p}<0.005)$ (Figure 3$)$.

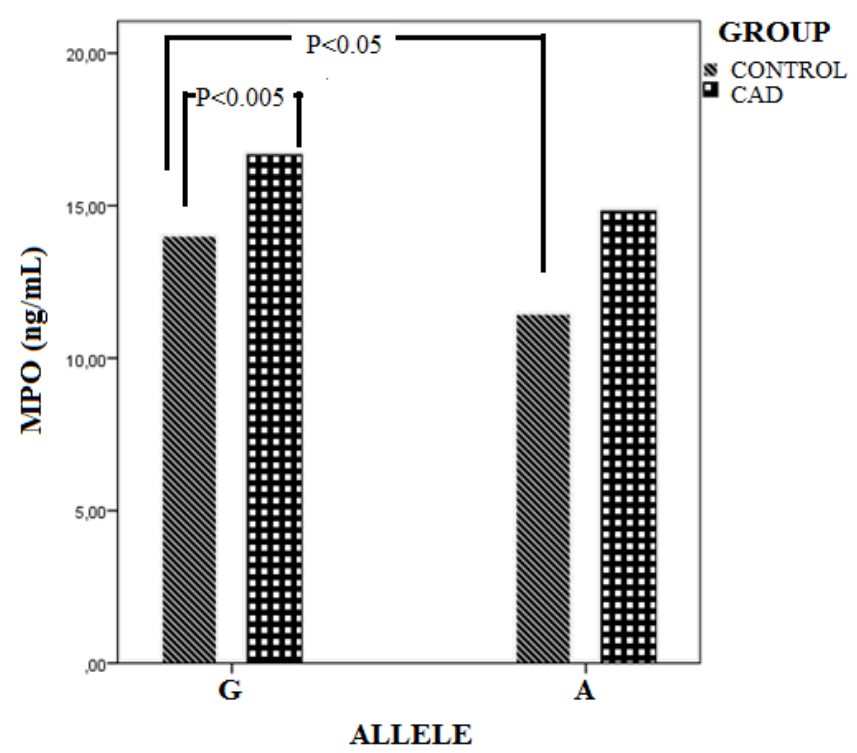

Figure 3. MPO levels in CAD patients and control subjects according to MPO alleles.

\section{Correlations between MPO and MMP-9 in total population}

Figure 4 shows the correlaitons between MPO and MMP-9 level in total population. There was a significant positive correlation between MPO and MMP-9 level $(r=0.243, \mathrm{P}=0.01)$.

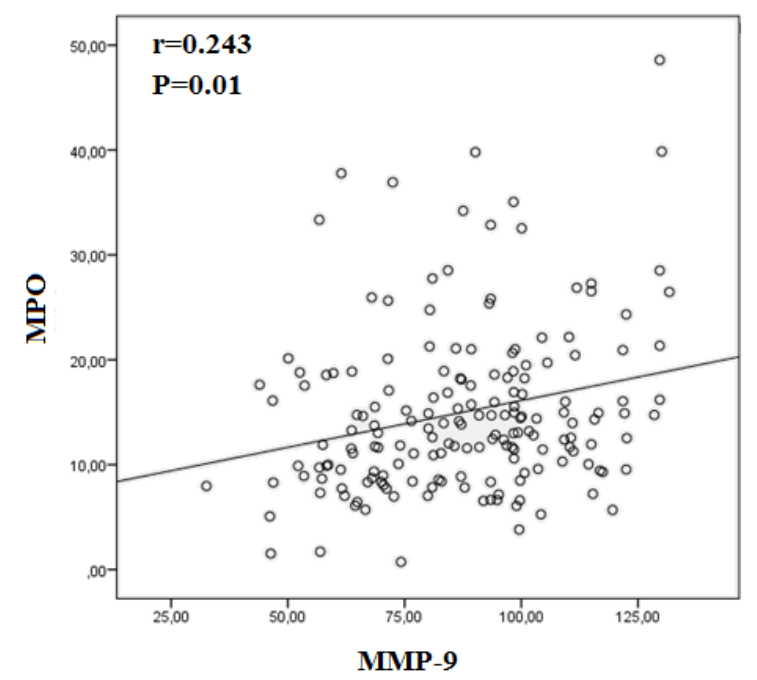

Figure 4. Correlations between MPO and MMP-9 in total population

\section{Discussion}

In this study we assessed plasma levels of MPO, MMP-9 and MPO gene polymorphism in CAD patients and control subjects in whom the presence of CAD was defined by coronary angiographic examination. The main findings of this study are as follows: (i) In patients with angiographically proven CAD, plasma MPO levels are elevated as compared with subjects without CAD; (ii) In patients with angiographically proven CAD, plasma MMP-9 levels are not difference as compared with subjects without CAD; (iii) We did not observed any significant differences between MPO genotypes and study groups and (iv) A positive correlation between MPO and MMP-9 level was found. 
CAD remains a heterogeneous disease with a wide range of clinical presentations and outcomes. Various systemic markers of inflammation have been investigated associated with CAD and cardiovascular events (26). The importance of PMN degranulation of MPO in the coronary circulation have emerged as powerful predictors of adverse outcome in patients with CAD (27-29). MPO is a redox-active hemoprotein released by activated neutrophils and is associated with inflammation and oxidative stress. Its role in catalyzing the formation of oxidizing agents. Hypochlorous acid, tyrosyl radical, and nitrogen dioxide are the products of MPO and they can contribute to oxidative damage of host lipids and proteins, leading to atherosclerosis. Brennan and colleagues (28) assessed the predictive value of serum MPO levels in patients with chest pain at a hospital emergency department. Serum MPO levels were significantly higher in patients who subsequently had a myocardial infarction (MI) within 16 hours of presentation (28). In a case-control study of 158 patients with CAD and 175 controls, Zhang et al. (11) have shown that elevated levels of blood MPO were associated with the presence of CAD. In the present study, we showed that corroborates the findings by Zhang et al. (11) in a series of patients in Turkey.

A few polymorphisms are described for the MPO gene, including the $-638 \mathrm{C} / \mathrm{A}$, the V53F and the $-463 \mathrm{~A} / \mathrm{G}$ (30). These polymorphisms could be genetically asociated with the pathophysiology of cardiovascular diseases. We chose the $-463 \mathrm{~A} / \mathrm{G}$ due to continuous literatures associated with coronary artery disease (13-16). Some studies have shown that GG genotype is associated with increased incidence of CAD (13-16), however, others failed to demonstrate a relation between MPO polymorphisms and atherosclerosis. We did not observed any significant differences between MPO genotypes and study groups. Furhermore, according to MPO -463 G/A allele in healthy groups with G allele had elevated MPO levels than A allele $(p<0.05)$ and subjects with $G$ alleles, CAD patients with $G$ genotype had higher level of MPO compared with controls $(\mathrm{p}<0.005)$.

Our study and the results have present certain limitations, since the study-group was obtained from a single hospital in East of Turkey, and the samples might not include all the characteristics of patients from other centers. It is also accepted that $\mathrm{CAD}$ is a disease effected by multiple genes and environmental factors. Furthermore, since the $M P O$ AA genotype is relatively infrequent, the actual value of genotype detection in the primary prevention of CAD may be disputable. However, to date, a number of studies have been conducted to assess the potential association between the MPO G-463A polymorphism and CAD. However the results were inconsistent.

MMPs had been identified in vascular remodeling process. They play an important role in the pathogenesis and progression of atherosclerosis (31). Several studies have shown the importance of these enzymes in development and progression of heart failure (32-34).

Some cross-sectional studies have reported that the plasma MMP-9 level was significantly higher in CAD patients $(35,36)$. Blankenberg et al. (2003) showed that plasma MMP-9 concentration was higher in patients with CAD and might be a novel predictor of cardiovascular disease (25). In the present study, we investigated the association of the MMP-9 levels with coronary artery disease. We did not observed any significant differences in MMP-9 levels between controls and CAD patients. However, some other cohort studies suggested that serum MMP-9 is not a strong predictor of CAD $(37,38)$. These inconsistent results can be explained as; plasma MMP-9 level is affected by many factors, such as genetic polymorphisms of MMP-9, drugs, and other factors. Lacchini et al. (2010) found that there are interethnic differences in MMP-9 genetic polymorphisms (39). furthermore, in our study, MPO levels were correlated with the increase in MMP-9 concentration $(P=0.01)$, supporting the previous mentioned hypothesis, that neutrophils could be the major contributors for increased MMP-9 levels.

\section{Conclusions}

Observations from this study suggest that MPO level was significantly higher in CAD patients but there was no significant association between MMP-9 level and CAD. We found that there was no significant differences between MPO genotypes and study groups. It is also generally accepted that $\mathrm{CAD}$ is a disease produced by both multiple genes and environmental factors, and many other genes could be potential candidates for CAD. Our results have present certain limitations, since the studygroup was obtained from a single hospital in east of Turkey, and the samples might not include all the characteristics of patients from other centers. However, further studies may be carried out in a larger population.

\section{Conflict of Interest}

The authors declare no conflicts of interests. 


\section{References}

1. Chisolm, G. M. \& Steinberg, D. The oxidative modification hypothesis of atherogenesis: An overview. Free Radic Biol Med 2000; 28: 1815-1826.

2. Wallenfeldt, K., Fagerberg, B., Wikstrand, J., \& Hulthe, J. Oxidized low-density lipoprotein in plasma is a prognostic marker of subclinical atherosclerosis development in clinically healthy men. J Intern Med 2004;256: 413-420

3. Tsimikas, S., Brilakis, E. S., Miller, E. R., Mcconnell, J. P., Lennon, R. J., Kornman, K. S., Witztum, J. L., \& Berger, P. B. () Oxidized phospholipids, Lp(a) lipoprotein, and coronary artery disease. $N$ Engl J Med 2005; 353, 46-57.

4. Stephens, J. W., Gable, D. R., Hurley, S. J., Miller, G. J., Cooper, J. A., \& Humphries, S. E. Increased plasma markers of oxidative stress are associated with coronary heart disease in males with diabetes mellitus and with 10 -year risk in a prospective sample of mailes. Clin Chem 2006;52: 446-453

5. Kathiresan, S., Srivastava, D. Genetics of human cardiovascular disease. Cell 2012;148: 12421257.

6. Stylianou, I.M., Bauer, R.C., Reilly, M.P., Rader, D.J.,. Genetic basis of atherosclerosis: insights from mice and humans. Circ. Res. 2012;110: 337-355.

7. Yusuf, S., Reddy, S., Ounpuu, S., Anand, S. Global burden of cardiovascular diseases:part I: general considerations, the epidemiologic transition, risk factors, and impact of urbanization. Circulation 2001;104: 2746-2753.

8. Zakynthinos E, Pappa N. Inflammatory biomarkers in coronary artery disease. J Cardiol. 2009;53(3):317-33.

9. Hoy A, Leininger-Muller B, Kutter D, Siest G, Visvikis S. Growing significance of myeloperoxidase in non-infectious diseases. Clin Chem Lab Med 2002;40:2- 8.

10. Tsimikas S. Oxidative biomarkers in the diagnosis and prognosis of cardiovascular disease. Am J Cardiol. 2006; 4;98(11A):9P-17P.

11. Zhang R, Brennan ML, Fu X, Aviles RJ, Pearce GL, Penn MS, Topol EJ, Sprecher DL, Hazen SL. Association between myeloperoxidase levels and risk of coronary artery disease. JAMA 2001;286:2136-2142.

12. Piedrafita FJ, Molander RB, Vansant G, Orlova EA, Pfahl M, Reynolds WF. An Alu element in the myeloperoxidase promoter contains a composite SP1-thyroidhormone-retinoic acid response element. J Biol Chem 1996;271:14412-20

13.Nikpoor B, Turecki G, Fournier C, Theroux P, Rouleau GA. A functional myeloperoxidase polymorphic variant is associated with coronary artery disease in French-Canadians. Am Heart $\mathbf{J}$ 2001;142:336-9.

14.Pecoits-Filho R, Stenvinkel P, Marchlewska A, et al. A functional variant of the myeloperoxidase gene is associated with cardiovascular disease in end-stage renal disease patients. Kidney Int Suppl 2003:S172-6.

15. Makela R, Laaksonen R, Janatuinen T, et al. Myeloperoxidase gene variation and coronary flow reserve in young healthy men. J Biomed Sci 2004;11:59-64. 
16. Asselbergs FW, Reynolds WF, Cohen-Tervaert JW, Jessurun GA, Tio RA. Myeloperoxidase polymorphism related to cardiovascular events in coronary artery disease. Am J Med 2004;116:429-30.

17. Mäkelä R, Karhunen PJ, Kunnas TA, et al. Myeloperoxidase gene variation as a determinant of atherosclerosis progression in the abdominal and thoracic aorta: an autopsy study. Lab Invest 2003;83:919-25.

18. Mäkelä R, Loimaala A, Nenonen A, et al. The association of myeloperoxidase promoter polymorphism with carotid atherosclerosis is abolished in patients with type 2 diabetes. Clin Biochem 2008;41:532-7.

19.Ye ZX ${ }^{1}$, Leu HB, Wu TC, Lin SJ, Chen JW. Baseline serum matrix metalloproteinase-9 level predicts long-term prognosis after coronary revascularizations in stable coronary artery disease. Clin Biochem. 2008;41(4-5):292-8.

20. Galis ZS, Sukhova GK, Lark MW, Libby P () Increased expression of matrix metalloproteinases and matrix degrading activity in vulnerable regions of human atherosclerotic plaques. J Clin Invest 1994;94: 2493-2503.

21. Loftus IM, Naylor AR, Goodall S, Crowther M, Jones L, et al. Increased matrix metalloproteinase- 9 activity in unstable carotid plaques. A potential role in acute plaque disruption. Stroke 2000;31: 40-47.

22. Nikkari ST, Hoyhtya M, Isola J, Nikkari T. Macrophages contain 92-kd gelatinase (MMP-9) at the site of degenerated internal elastic lamina in temporal arteritis. Am J Pathol 1996;149: $1427-1433$.

23. Brown DL, Hibbs MS, Kearney M, Loushin C, Isner JM. Identification of 92-kD gelatinase in human coronary atherosclerotic lesions. Association of active enzyme synthesis with unstable angina. Circulation 1995;91: 2125-2131.

24. Ferroni P, Basili S, Martini F, et al. Serum metalloproteinase 9 levels in patients with coronary artery disease: a novel marker of inflammation. J Investig Med 2003;51:295-300.

25.Blankenberg S, Rupprecht HJ, Poirier O, et al. Plasma concentrations and genetic variation of matrix metalloproteinase 9 and prognosis of patients with cardiovascular disease. Circulation 2003; 107:1579-85.

26. Fichtlscherer S, Heeschen C, Zeiher AM. Inflammatory markers and coronary artery disease. Curr Opin Pharmacol 2004;4:124-31.

27. Baldus S, Heeschen C, Meinertz T, et al. Myeloperoxidase serum levels predict risk in patients with acute coronary syndromes. Circulation 2003;108:1440-5.

28.Brennan ML, Penn MS, Van Lente F, et al. Prognostic value of myeloperoxidase in patients with chest pain. N Engl J Med 2003;349:1595-604.

29. Cavusoglu E, Ruwende C, Eng C, et al. Usefulness of baseline plasma myeloperoxidase levels as an independent predictor ofmyocardial infarction at two years in patients presenting with acute coronary syndrome. Am J Cardiol 2007;99:1364-8.

30. Chevrier I, Tregouet DA, Massonnet-Castel S, Beaune P, Loriot MA. Myeloperoxidase genetic polymorphisms modulate human neutrophil enzyme activity: genetic determinants for atherosclerosis? Atherosclerosis 2006;188:150-4.

31. Sundstrom J, Vasan RS. Circulating biomarkers of extracellular matrix remodeling and risk of atherosclerotic events. Curr Opin Lipidol 2006;17:45-53. 
32. Thomas CV, Coker ML, Zellner JL, Handy JR, Crumbley III JC, Spinale FG. Increased matrix metalloproteinases activity and selective upregulation in left ventricular myocardium from patients with end-stage dilated cardiomyopathy. Circulation 1998;97:1708-15.

33. Spinale FG, Coker ML, Krombach SR, Mukherjee R, Hallak H, Houck WV, et al. Matrix metalloproteinase inhibition during the development of congestive heart failure. Effects on left ventricular dimensions and function. Cir Res 1999;85:364-76.

34. Peterson JT, Dillon L, Bryant JW. Evolution of metalloproteinase and tissue inhibitor expression during heart failure progression in the infracted heart. Cardiovasc Res 2000;46:30715.

35. Kai, H., Ikeda, H., Yasukawa, H., et al. Peripheral blood levels of matrix metalloproteases-2 and -9 are elevated in patients with acute coronary syndromes. J. Am. Coll. Cardiol.1998;32(2):368-372.

36. Tayebjee, M.H., Lip, G.Y., Tan, K.T., et al. Plasma matrix metalloproteinase-9, tissue inhibitor of metalloproteinase-2, and CD40 ligand levels in patients with stable coronary artery disease. Am. J. Cardiol., 2005;96(3): 339-345. [doi:10.1016/j.amjcard.2005.03.072]

37. Jefferis, B.J., Whincup, P., Welsh, P., Wannamethee, G., Rumley, A., Lennon, L., Thomson, A., Lawlor, D., Carson, C., Ebrahim, S., Lowe, G. Prospective study of matrix metalloproteinase-9 and risk of myocardial infarction and stroke in older men and women. Atherosclerosis 2010;208: $557-563$.

38. Welsh, P., Whincup, P.H., Papacosta, O., et al.,Serum matrix metalloproteinase-9 and coronary heart disease: a prospective study in middle-aged men. QJM, 2008;101(10): 785-791. [doi:10.1093/qjmed/hcn088]

39. Lacchini, R., Metzger, I.F., Luizon, M., et al. Interethnic differences in the distribution of matrix metalloproteinases genetic polymorphisms are consistent with interethnic differences in disease prevalence. DNA Cell Biol. 2010; 29(11):649-655. [doi:10.1089/dna.2010.1056] 